\title{
Giant gastric lipoma presenting with melaena: A case report and review of the literature
}

\author{
Vishwanath Vijay Joshi', Neemish Kamat ${ }^{2}$, Saumya Rao ${ }^{3}$, Deepak Patkar ${ }^{4}$ \\ From ${ }^{1}$ Fellow, ${ }^{2}$ Consultant and Head, ${ }^{3}$ Resident, ${ }^{4} \mathrm{Head}$, Department of Radiology, Dr. Balabhai Nanavati Hospital, Mumbai, Maharashtra, India
}

\begin{abstract}
Gastric lipomas are rare tumors of the gastrointestinal tract. The majority of the tumors are asymptomatic and detected incidentally. However, large tumors can present either bleeding or gastric outlet obstruction. Here, we present a case of giant gastric lipoma in the pyloric antrum presenting with melaena and treated by surgical resection. An elderly male presented to the emergency department with a history of abdominal pain and melanotic stools. Computed tomography (CT) showed a homogeneous submucosal mass of fat attenuation in the gastric antrum with ulceration subsequently confirmed on endoscopy. The patient underwent surgical resection of the tumor with distal gastrectomy. Pathologic examination showed a well-circumscribed, encapsulated mass comprised mature adipose tissue without atypia or mitotic figures, consistent with lipoma. This case highlights the importance of $\mathrm{CT}$ in not only predicting the accurate diagnosis of these masses but also to provide additional details such as sarcomatous changes and perigastric spread before endoscopy or surgery.
\end{abstract}

Key words: Computed tomography, gastric lipoma, melaena

\section{A} gastric lipoma is a rare lesion, accounting for only $5 \%$ of gastrointestinal tract lipomas and less than $1 \%$ of all gastric tumors [1]. Most gastric lipomas are found in the submucosa of the pyloric antrum. The majority of these are asymptomatic. Bleeding due to mucosal erosions on top of the lipomas and mass effect leading to gastric outlet obstruction is some of the common presentations symptomatic individuals. We present a case of giant gastric lipoma in an elderly individual presenting with multiple episodes of melaena.

\section{CASE REPORT}

A 60-year-old male presented to the emergency department with complaints of abdominal pain and multiple episodes of melaena. The pain was non-colicky type and associated with the passage of stools. The history was remarkable for similar such episodes at least 4-5 times for the past 1 year and was treated conservatively with blood transfusions on two occasions. There was no history of the patient on aspirin or any other blood thinners.

Vital parameters of the patient were normal with pulse rate 76 beats/min, blood pressure $-130 / 80 \mathrm{mmHg}$, and $\mathrm{SpO}_{2}-96 \%$ at room air. There was evidence of anemia on general examination with the rest of the systemic examination within normal limits.

\section{Access this article online}

Received - 01 September 2020 Initial Review - 17 September 2020 Accepted - 26 September 2020

DOI: $10.32677 /$ IJCR.2020.v06.i10.002
The local examination was remarkable for epigastric tenderness with no palpable mass lesion.

Laboratory findings were as follows: Hemoglobin: $8.9 \mathrm{~g} / \mathrm{dL}$, mild leukocytosis $17,450 / \mathrm{mm}^{3}$, platelet count: $415,000 / \mathrm{mm}^{3}$ with prothrombin time: 10.5 seconds, and international normalized ratio of 0.95 . Liver and kidney function tests were normal. The patient was referred to the radiology department for computed tomography (CT) scan of the abdomen and pelvis with angiography to rule out the cause of bleeding. CT demonstrated a fairly large well-defined hypoattenuating lesion in the submucosa of the pyloric antrum of the stomach measuring about $5.2 \mathrm{~cm} \times$ $7.9 \mathrm{~cm}$ in maximal axial dimension and a cephalocaudal extent of about 5.5 to $6.5 \mathrm{~cm}$ exhibiting attenuation values of adipose tissue ( $-38--70 \mathrm{HU})$ cephalad projecting into the distal body of the stomach and caudally into the D1 segment of the duodenum (Fig. $1 \mathrm{a}-\mathrm{c}$ ). Areas of ulceration with pockets of air are seen along the greater curvature and inferior margin of the lesion (Fig. 1d).

No evidence of active extravasation of contrast was seen. Few twigs of vessels from the left gastric artery were seen to supply the lesion. There were focal uniform thickening and mild accentuated enhancement of the walls of the pyloric antrum which measures about 5-7 $\mathrm{mm}$. This lesion causes virtual total luminal compromise with no gastric outlet obstruction. There was no stranding of the perigastric fat or significant perigastric adenopathy. These CT findings were suggestive of a large submucous lipoma in the

Correspondence to: Dr. Vishwanath Vijay Joshi, Department of Radiology, Dr. Balabhai Nanavati Hospital, Vile Parle, Mumbai - 400 056, Maharashtra, India. E-mail: vishuvjoshi@gmail.com

(C) 2020 The Author(s). This open access article is distributed under a Creative Commons Attribution (CC-BY) 4.0 license. 

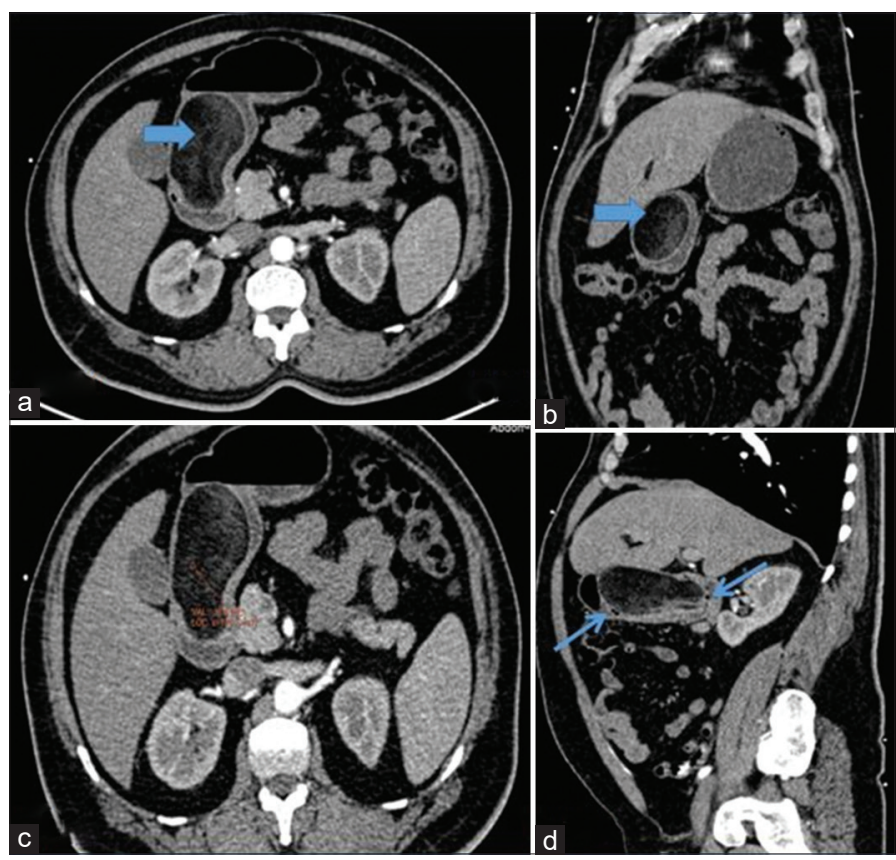

Figure 1: (a-d) Computed tomography showing well-defined hypoattenuating lesion in the submucosa of the pyloric antrum of the stomach (thick arrows) exhibiting attenuation values of adipose tissue $(-38--70 \mathrm{HU})$ cephalad projecting into the distal body of the stomach and caudally into the D1 segment of the duodenum. Areas of ulceration with pockets of air (thin arrows) seen along greater curvature and inferior margin of the lesion

pyloric antrum of the stomach. The patient then underwent upper gastrointestinal endoscopy for further evaluation of the mass. A small hiatus hernia was seen. The large subepithelial mass lesion extending from the incisura to the pyloric channel was seen with one deep ulcer and two small ulcers adjacent to it (Fig. 2). There was no evidence of any active bleed.

In view of the large lesion and recurrent symptoms, surgical resection of the tumor was done. Exploratory laparotomy with distal gastrectomy and retrocolic loop gastrojejunostomy was performed. Infracolic jejunojejunostomy was done for direct drainage of bile along with feeding jejunostomy. Corrugated drain placed at the duodenal stump and below gastrojejunostomy with hemostasis achieved.

Pathological examination showed a large submucosal polypoidal mass measuring $9.5 \mathrm{~cm} \times 7 \mathrm{~cm} \times 4 \mathrm{~cm}$. The cut surface was yellowish-white, smooth, and lobulated. The mass shows ulceration measuring $2 \mathrm{~cm} \times 1.5 \mathrm{~cm}$. Microscopy showing an encapsulated adipocytic tumor composed of mature adipocytes arranged in lobules and separated by fibrous septae. The adipocytes are of uniform shape and size with no lipoblasts (Fig. 3). Adjacent mucosal shows chronic gastritis with thickening of muscularis mucosae. The above features were consistent with gastric submucosal lipoma. The postoperative period was uneventful and the patient is presently on routine follow-up with no symptoms present.

\section{DISCUSSION}

Lipomas are slow-growing, benign, and fatty tumors enclosed by a thin fibrous capsule and are the most commonly encountered

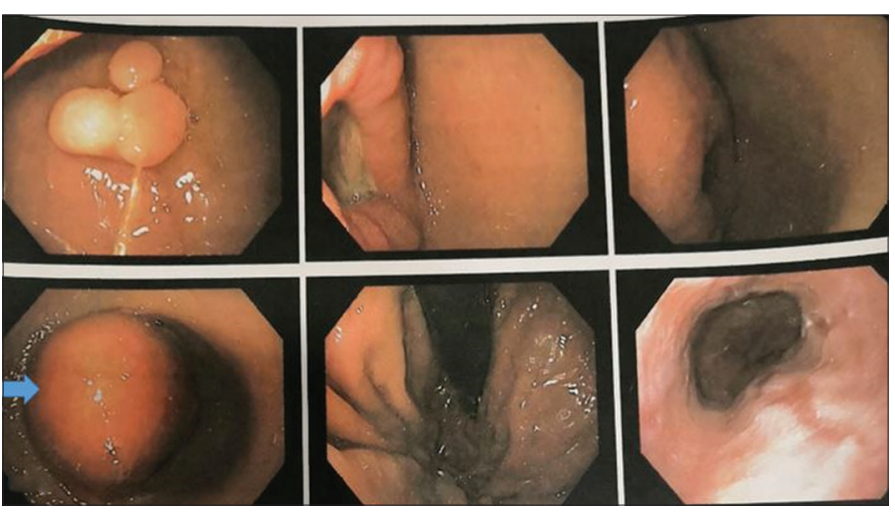

Figure 2: Upper GI endoscopy showing a large subepithelial mass lesion extending from incisura to pyloric channel with no evidence of any active bleed

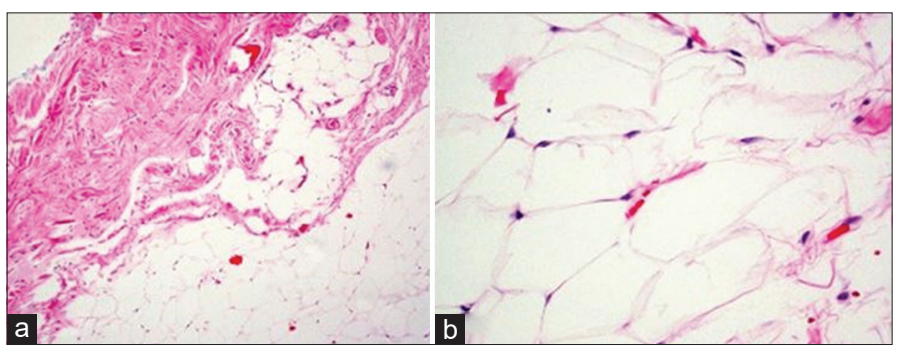

Figure 3: ( a and b) Microscopy showing adipocytic tumor composed of mature adipocytes arranged in lobules and separated by fibrous septae

soft-tissue tumors. They are derived from the mesenchymal origin and develop in virtually all organs throughout the body, including the gastrointestinal tract, where they present as submucosal fatty tumors. The most common gastrointestinal tract locations for lipoma are the colon, ileum, and jejunum [2]. Gastric lipomas are rare and represent $3 \%$ of benign gastric masses. Only $5 \%$ of alimentary tract lipomas occur in the stomach, the second rarest location after the esophagus [3]. These tumors tend to occur in individuals aged 40-50 years with no sex predilection [4]. There are no known risk factors for the development of gastric lipoma, but have been observed to occur at a higher rate in obese individuals and in diabetic patients [5]. Our case was an obese diabetic individual with an age of 60 years.

The pathophysiology of gastric lipomas is not entirely understood. Numerous etiologies have been hypothesized, including embryonic misplacement of adipose tissue precursors, chronic irritation, and low-grade infection [6]. Histologically gastric lipomas do not differ from lipomas found in other regions of the body and are composed of mature fat surrounded by a fibrous capsule, characteristics that are demonstrated on CT. Differential diagnosis includes gastric liposarcomas, which very rare and characterized by the locally aggressive appearance and propensity for distant metastases.

The majority of gastric lipomas are asymptomatic. If symptomatic, upper gastrointestinal bleeding secondary to mucosal ulceration (50-60\%) or obstructive symptoms due to pedunculated lesions are most common [7]. On physical examination, these lesions can present as fullness in the epigastrium, as a distinct, palpable mass, or with a normal examination. 
On radiographs, a lipoma can occasionally appear as a radiolucent shadow. During fluoroscopy, a lipoma appears as a smooth well-marginated mass, sometimes with central ulceration. Because lipomas are soft tumors, they can change in size and shape with peristalsis or palpation during fluoroscopy [8].

On ultrasound, gastric lipomas appear as homogenously hyperechoic masses marginated by a fibrous hypoechoic capsule [9]. However, the appearance may be variegated or hypoechoic in lesions associated with hemorrhage or those associated copious submucosal fat. Endoscopic ultrasound (EUS) is recommended for biopsy and resection planning as it allows better localization of the lesion within five gastric layers [10].

On CT, gastric lipomas appear as round or ovoid masses with low attenuation, homogenous appearance, measuring between -80 and -120 Hounsfield units. These findings are nearly pathognomonic. If there is a presence of linear strands of soft-tissue attenuation, this may represent associated ulcer and fibrovascular septa but should raise liposarcoma on the differential $[2,11]$. Thompson et al., in their review, our review of data for 16 patients with proven gastric lipomas, revealed two significant findings. First was on upper gastrointestinal examinations, lipomas have the appearance of any submucosal tumor and are indistinguishable from gastrointestinal stroma tumors or lymphoma. Second, CT findings are specific for the diagnosis of gastric lipoma. CT should be used to characterize large submucosal masses before an endoscopic biopsy is performed [11]. CT has both superior sensitivity and specificity for the diagnosis of gastric lipoma compared to fluoroscopy, endoscopy, and EUS [12]. Gastric liposarcomas are exceedingly rare. On CT, these usually appear heterogeneous with soft-tissue density and cystic areas suggestive of necrosis and hemorrhage [13].

Magnetic resonance imaging (MRI) is a good alternative in individuals with a risk of radiation exposure like in the pediatric population. On MRI, gastric lipomas demonstrate isointensity to fat on T1- and T2-weighted imaging with decreased signal on fat suppression and out of phase MR sequences [14]. On esophagogastroduodenoscopy, these lesions appear as soft, sharply defined, submucosal masses, often yellow in color as opposed to the surrounding pink mucosa. Several signs, such as tenting sign, cushion sign, and naked fat sign, have been described to help identify lipomas on endoscopic examination [15]. Sullivan et al., in their review, investigated the multimodality imaging findings of gastric lipomas. The antrum was the most common location in their case series, as was in the present case. They concluded that CT is the diagnostic gold standard, surpassing other imaging studies, endoscopy, and even routine endoscopic biopsy in both sensitivity and specificity [16].

This case report illustrates a rare case of symptomatic gastric lipoma presenting with melaena with a review of relevant literature. The pivotal role of CT in diagnosing these tumors and also associated complications has been discussed.

\section{CONCLUSION}

Giant gastric lipomas are rare tumors of the stomach with CT being the best modality for the diagnosis exhibiting attenuation values of $-70-120 \mathrm{HU}$. Large symptomatic lipomas presenting with either bleeding or obstruction need prompt diagnosis and intervention in the form of endoscopic or surgical resection.

\section{REFERENCES}

1. Fernandez MJ, Davis RP, Nora PF. Gastrointestinal lipomas. Arch Surg 1983;118:1081-3.

2. Taylor AJ, Stewart ET, Dodds WJ. Gastrointestinal lipomas: A radiologic and pathologic review. AJR Am J Roentgenol 1990;155:1205-10.

3. Chu AG, Clifton JA. Gastric lipoma presenting as peptic ulcer: Case report and review of the literature. Am J Gastroenterol 1983;78:615-8.

4. Hamdane MM, Brahim EB, Salah MB, Haouas N, Bouhafa A, ChedlyDebbiche A. Giant gastric lipoma mimicking well-differentiated liposarcoma. Gastroenterol Hepatol Bed Bench 2012;5:60-3.

5. Goldblum JR, Folpe AL, Weiss SW. Benign lipomatous tumors. In: Enzinger and Weiss's Soft Tissue Tumors. Ch. 14. Philadelphia, PA: Mosby Elsevier; 2018. p. 443-83.

6. Weinberg T, Feldman M. Lipomas of the gastrointestinal tract. Am J Clin Pathol 1955;25:272-81.

7. Nickloes TA, Sutphin DD. Lipomas: Background, Pathophysiology and Etiology. Medscape; 2017. Available from: https://www.emedicine. medscape.com/article/191233. [Last accessed on 2017 Dec 15].

8. Kang HC, Menias CO, Gaballah AH, Shroff S, Taggart MW, Garg N, et al. Beyond the GIST: Mesenchymal tumors of the stomach. Radiographics 2013;33:1673-90.

9. Regge D, Lo Bello G, Martincich L, Bianchi G, Cuomo G, Suriani R, et al. A case of bleeding gastric lipoma: US, CT and MR findings. Eur Radiol 1999;9:256-8.

10. Kim CY, Bandres D, Tio TL, Benjamin SB, Al-Kawas FH. Endoscopic removal of large colonic lipomas. Gastrointest Endosc 2002;55:929-31.

11. Thompson WM, Kende AI, Levy AD. Imaging characteristics of gastric lipomas in 16 adult and pediatric patients. AJR Am J Roentgenol 2003;181:891-985.

12. Tang SY, Huang Y, Zhang CL, Cheng C, Kuang LQ, Wang Y. Comparative study of MSCT, endoscopy and gastrointestinal tract radiography in diagnosis of gastric lipomas. Med J Chin PLA 2017;42:154-7.

13. Elhjouji A, Jaiteh L, Mahfoud T, Belhamidi S, Bounaim A, AitAli A, et al. Giant gastric liposarcoma: A fatal exceptional location. J Gastrointest Cancer 2016;47:482-5.

14. Richman DM, Tirumani SH, Hornick JL, Fuchs CS, Howard S, Krajewski K, et al. Beyond gastric adenocarcinoma: Multimodality assessment of common and uncommon gastric neoplasms. Abdom Radiol (NY) 2017;42:124-40.

15. Amundson JR, Straus D, Azab B, Liu S, Buitrago MT, Yakoub D. Giant symptomatic gastric lipoma: A case report and literature review. Int J Surg Case Rep 2018;51:313-7.

16. Sullivan IW, Hota P, Dass C. Gastric lipomas: A case series and review of a rare tumor. BJR Case Rep 2019;5:20180109.

Funding: None; Conflicts of Interest: None Stated.

How to cite this article: Joshi VV, Kamat N, Rao S, Patkar D. Giant gastric lipoma presenting with melaena: A case report and review of the literature. Indian J Case Reports. 2020;6(10):550-552. 